\title{
Prioritizing barriers to implement clinical governance in teaching hospitals of Iran: A mixed method study
}

\author{
Abbas Ziari ${ }^{1}$, Maryam Rassouli ${ }^{2}$, Fariba Mirbaha-Hashemi ${ }^{3}$, Mohammad Ali Heidarnia ${ }^{4}$, \\ Kambiz Abachizadeh*4,5
}

Received: 29 Jun 2017

Published: 18 Dec 2019

\section{Abstract}

Background: Clinical governance, as a program to improve the quality of health care, was introduced in all hospitals in Iran in 2009. However, implementation assessments revealed that the program was not adopted in many hospitals. This study aimed to determine and prioritize barriers to clinical governance program implementation in hospitals of Shahid Beheshti University of Medical Sciences.

Methods: This qualitative study was performed in the first part of this exploratory mixed methods study. A purposive sample of 25 individuals who were involved in the implementation of the program was selected. They participated in semi-structured interviews and the data were analyzed using content analysis. In the second part (quantitative), a questionnaire was prepared based on the first phase of the study, and 74 stakeholders completed the questionnaire, which included all extracted obstacles to the establishment of clinical governance. They rated these obstacles with a 5-point Likert scale.

Results: A total of 9 themes were discovered in the qualitative part of the study. These themes were prioritized as follow: (1) weak organizational leadership, (2) insufficient human resources, (3) inappropriate organizational culture, (4) inadequate financial resources, (5) insufficient knowledge of personnel and management, (6) inappropriate monitoring and evaluation, (7) lack of coordination (8) deficiencies in policies and procedures, and (9) incomplete registration system and inadequate documentation.

Conclusion: The results of this study showed that significant barriers exist in implementation of the clinical governance program in hospitals. These problems have to be addressed in order for the implementation procedure to be successful.

Keywords: Hospital, Clinical governance, Qualitative study, Barrier, Prioritize

Conflicts of Interest: None declared

Funding: None

*This work has been published under CC BY-NC-SA 1.0 license.

Copyright $\odot$ Iran University of Medical Sciences

Cite this article as: Ziari A, Rassouli M, Mirbaha-Hashemi F, Heidarnia MA, Abachizadeh K. Prioritizing barriers to implement clinical governance in teaching hospitals of Iran: A mixed method study. Med J Islam Repub Iran. 2019 (18 Dec);33:140. https://doi.org/10.47176/mjiri.33.140

\section{Introduction}

Various approaches for improving the quality of health care services have been adopted by different countries. Clinical governance is one of these approaches. This program was first proposed by the United Kingdom state health system as a strategy to improve the quality of clinical care in 1998 (1). Clinical governance is based on 7 pillars,

Corresponding author: Dr Kambiz Abachizadeh, k.abachizadeh@sbmu.ac.ir

1. Social Determinants of Health Research Center, Semnan University of Medical Sciences, Semnan, Iran

2. Cancer Research Center, Shahid Beheshti University of Medical Sciences, Tehran, Iran

3. Preventive Medicine and Public Health Research Center, Iran University of Medical Sciences, Tehran, Iran

4. Department of Community Medicine, School of Medicine, Shahid Beheshti University of Medical Sciences, Tehran, Iran

5. Social Determinants of Health Research Center, Shahid Beheshti University of Medical Sciences, Tehran, Iran including clinical effectiveness, risk management, patient and public involvement, clinical information, education and training, clinical audit, and staffing and staff management (2).

In the clinical governance framework, health care provid-

$\uparrow$ What is "already known" in this topic:

There is little information on how clinical governance program has been conducted in Iranian hospitals.

\section{$\rightarrow$ What this article adds:}

Main barriers to implement clinical governance program in investigated hospitals in order of importance included weak organizational leadership, insufficient human resources, inappropriate organizational culture, inadequate financial resources, and insufficient knowledge of personnel and the management, inappropriate monitoring and evaluation, and lack of coordination among various departments in the organization. 
ers are held accountable for continuing to improve the quality of their services. As the accountability increases, the consistency in the clinical service delivery also improves. Moreover, one of the main principles of clinical governance is proper handling of medical errors to eliminate inappropriate care (3).

The global widespread shift and focus of health systems on quality of care prompted Ministry of Health and Medical Education (MOHME) of Iran to adopt Clinical Governance as a policy to be implemented in all hospitals across the country. In November 2009, under document number 388044, MOHME introduced clinical governance to the hospitals to standardize and improve clinical services, increase efficiency, and reduce costs. In order to achieve the goals of this program, clinical governance offices were established in all universities and hospitals in 2011, including Shahid Beheshti University of Medical Sciences in Tehran $(4,5)$.

Since the implementation of clinical governance in Iran, some studies have been conducted to identify the barriers and challenges to its utilization. The results of these studies show diverse challenges and problems that still exist in the implementation of this policy.

Despite all efforts, service quality and quality improvement measures have shown to be far from what is expected. Collecting adequate countrywide information about the quality of service is essential (6).

Further, a study that evaluated the progress of clinical governance implementation in one of the teaching hospitals in Tehran revealed that clinical effectiveness and clinical audit have not improved in that hospital (7).

Moreover, in a study in Mashhad Medical School hospitals in east of Iran, the main barriers to implement clinical governance were identified as incompetent hospital administrators, inadequate human resources, improper use of human resources, insufficient knowledge of clinical staff about clinical governance, lack of appropriate incentives, and inappropriate organizational culture (8). Other problems in the implementation process include lack of an integrated comprehensive implementation plan, lack of a proper notification and training systems about clinical governance, incompetence in implementation, and inadequate attention to important needs of health care centers and patients (9).

Noticeably, the role of physicians in the establishment and implementation of clinical governance is highly important. A study showed that the highest resistance to changes made by the program was from the physician (10). Another study revealed that, in the mid-level administrators, the most important challenge in implementing the program was their lack of clear understanding of the basic concepts of clinical governance (11).

Despite the need for implementation of clinical governance in all hospitals around the country and requirement of the MOHME for its complete and effective establishment, only a few studies have been done and no exploratory mixed method study has been performed to identify barriers for effective implementation of the program. In addition, barriers have not been prioritized in the previous studies. By identifying priorities, obstacles that are more basic and essential can be considered as the primary focus. Therefore, this study aimed to determine the barriers of establishing clinical governance and prioritizing these problems in the teaching hospitals of Shahid Beheshti University of Medical Sciences.

\section{Methods}

This study was an exploratory sequential mixed methods research which included a qualitative and a quantitative phase.

\section{Qualitative study}

Participant selection: Participants in this study comprised of the main stakeholders in the implementation of clinical governance, including physicians, nurses, hospital managers, experts of clinical governance, clinical directors of Shahid Beheshti University of Medical Sciences and directors of Ministry of Health. These individuals were chosen from 3 implementation layers, including the teaching hospitals of the university, clinical administration of the university, and the Ministry of Health. The participants in the study were selected by purposive sampling because of their knowledge, experience, and expertise in the implementation of clinical governance. Informed consent was obtained from each participant in the study.

Data collection: Data were collected using semistructured interviews guided based on content analysis approach (12).

The questions used in the interviews were reviewed and approved by 9 qualitative researchers with specialization in health care management, health care policy, or nursing. A total of 25 interviews were conducted until data saturation. All interviews were done by the first author. They were conducted in the participants' place of work with prior arrangements. Some of the interview questions were as follow:

- What do you think about the establishment of clinical governance in hospitals affiliated to medical universities?

- Please tell me about your experience of the implementation process of the program?

- Did you ever feel that the implementation process was slowed down or stopped? If yes, in your opinion, what were the reasons?

Interviews lasted from 55 to 84 minutes. They were recorded with permission from the participants and were transcribed verbatim as soon as possible after the interviews. The transcription texts were compared with the recordings to confirm their accuracy.

\section{Data Analysis}

Data were analyzed according to the content analysis approach of Graneheim and Lundman (13). That is, meaning units from interview texts were extracted and coded. Then, interview number was inserted next to each of the coded comments. In the next step, similar codes were grouped and primary categories were formed. The highest homogeneity was maintained within the categories and the most heterogeneity was between the categories. Next, the categories 
were named. In the next step, these categories were reviewed again to extract the main themes, which revealed the most important problems and barriers for implementation of clinical governance in teaching hospitals.

\section{Data trustworthiness}

Trustworthiness (credibility, transferability, dependability, and confirmability) was achieved through data source triangulation (use of experts with various backgrounds, specializations, and occupations in the collection of data). All participants had experience with implementation of clinical governance and were willing to talk about their experiences. Researchers' assumptions were identified at the beginning of research to prevent possible impacts on the data analysis. Three researchers extracted the codes and categories to ensure consistency of the results with the interview texts (14).

\section{Quantitative study}

In the quantitative phase, a questionnaire was prepared based on the extracted themes in the qualitative phase, which actually revealed the barriers to implementation of clinical governance. The questionnaire was emailed to 89 experts, and 78 (or $87.6 \%$ ) completed the questionnaire and returned them. These individuals had knowledge, experience, and expertise on the subject matter and included nurses, hospital supervisors, managers and directors, clinical directors, and experts of the Ministry of Health. They were selected through purposive sampling. They were asked to rate each item on the questionnaire for importance, on a Likert scale of 1 to 5 ( 1 for the least important and 5 for the most important). The questionnaires were emailed and followed-up by a phone call. To determine the priority of the barriers, average scores were calculated based on the participants' responses and a prioritized list of barriers was then prepared accordingly.

\section{Ethical considerations}

Prior to each interview, written consent was obtained from the participants after explaining the study objectives, confidentiality of data, anonymous publication of the results, and the right to withdraw at any stage of the interview. Ethical principles and respect for participants' rights were observed in the course of each and every interview.

\section{Results}

\section{Qualitative study}

Of the 25 participants, 13 (52\%) were women and 12 (48\%) were men and aged 35-57 years. Their fields of study included nursing, health education, library science, public administration, and health care management. General practitioners and specialist physicians were also included. They had 10-23 years of experience in their occupation.

A total of 2956 codes were extracted from analysis of all interviews and were placed in 30 categories, some of which were merged together based on their meaning and concept. Finally, 9 main themes were extracted (Table 1).

The identified themes or barriers, in order of importance, along with some of the participants' comments are as follow:

\section{Weak organizational leadership}

Almost all participants in this study mentioned the importance of leadership. They pointed out that one of the main barriers to the implementation of clinical governance was a lack of attention to proper timing and a step-by-step timetable. A participant (43 years old with $\mathrm{Ph} . \mathrm{D}$. in health

Table 1. Themes and categories of the obstacles and barriers to implement clinical governance

\begin{tabular}{|c|c|c|}
\hline Themes & Categories & \\
\hline \multirow[t]{5}{*}{ Insufficient human resources } & $>$ & Insufficient manpower \\
\hline & $>$ & Education \\
\hline & & Experience \\
\hline & & Inefficient performance \\
\hline & $>$ & Incompetence of the manpower \\
\hline \multirow[t]{2}{*}{ Inadequate financial resources } & $>$ & Inadequate funding \\
\hline & & Independent budget allocation challenges \\
\hline \multirow{3}{*}{$\begin{array}{l}\text { Incomplete registration system and inadequate documenta- } \\
\text { tion }\end{array}$} & $>$ & Infrastructure \\
\hline & $>$ & Hospital Information System (HIS) \\
\hline & & Inadequate facilities \\
\hline \multirow[t]{5}{*}{ Inappropriate organizational culture } & $>$ & poor planning \\
\hline & & Unrealistic expectations \\
\hline & & Frequent change of management \\
\hline & $>$ & Inadequate use of national and international experiences \\
\hline & $>$ & Inappropriate plan for the current situation and existing facilities \\
\hline Insufficient knowledge of personnel and the management & $>$ & Insufficient or lack of training of staff and the management \\
\hline \multirow[t]{4}{*}{ Deficiencies in policies and procedures } & $>$ & Lack of reinforcement of guidelines and policies \\
\hline & & Need for proper documentations \\
\hline & & Need for specific and standard procedures and policies \\
\hline & $>$ & Inadequate standards to optimize the manpower \\
\hline Inappropriate monitoring & $>$ & Need for standard performance-based indicators \\
\hline \multirow[t]{2}{*}{ and evaluation } & $>$ & Need for standard and adequate monitoring and evaluation \\
\hline & $>$ & Need for professional monitoring and evaluation experts \\
\hline \multirow{2}{*}{$\begin{array}{l}\text { Lack of coordination among various departments in the or- } \\
\text { ganization }\end{array}$} & & Lack of Integration and coordination among all systems in the hospital \\
\hline & $>$ & $\begin{array}{l}\text { Simultaneous implementation of several different programs with dif- } \\
\text { ferent goals }\end{array}$ \\
\hline \multirow[t]{3}{*}{ Weak organizational leadership } & $>$ & Inadequate planning and timetables \\
\hline & & Need for full commitment of the management \\
\hline & $>$ & Rushing into the implementation of the program \\
\hline
\end{tabular}


care management) stated, "The problem is that all of a sudden there comes a program which was designed outside the hospital and it has to be launched immediately... The staff is not familiar with it, there is no step by step plan, and it all has to be implemented immediately while the departments have no say in the matter, and all this is for a program that requires a lot of time and effort just to grasp its concept...."

All participants stressed the importance of top management commitment and also top and mid-level management's willingness to follow-up the implementation progress of the program. One participant (42 years old with Ph.D. in health administration) said, "Wherever managers and officials showed willingness, the program was implemented and followed-up actively. However, wherever a manager had no interest in the program, the program was trimmed and only a bunch of written procedures in a binder was left of it.... The roles of the officials and managers at the hospital and also ministerial levels are very influential and significant."

\section{Insufficient human resources}

Most of participants in this study cited inadequate human resources and lack of sufficient motivation and ability as major problems. In this regard, one participant (49 years old with Master's degree in public administration) stated, "For example, in the emergency department, once for a meeting about audit, whoever was called to attend the meeting was busy somewhere else and was not available, so the meeting was canceled." Most participants stated that there was an urgent need for sufficient and capable human resources. One of the participants (a 49-year-old nurse) mentioned, "A lot of times, the person responsible for following-up the program has other responsibilities in the hospital and is unable to pay careful attention to everything. Because they did not have enough personnel, they had to make that particular person responsible but that person has no motivation." A 45 years old supervisor said, "Unfortunately, those people who are supposed to monitor the implementation of the program and be responsible for it, are not capable and powerful individuals; as a result, the program is not progressing well."

\section{Inappropriate organizational culture}

The participants in this study mentioned that to implement program properly, available experience inside and outside of the country, current situation, and existing facilities should be taken into consideration to make a plan appropriate for this country. Furthermore, cultural aspects should be taken into account during the planning phase.

A 47 years old supervisor noted, "When changes are made in the management from time to time, the new directors do not care about the program. Basically, in our organizational culture, when an administrator changes, all programs experience drastic changes, while the programs should not be dependent on an individual." On the other hand, because of the lack of a systematic organizational culture, some unrealistic expectations lead to hasty decisions which may cause frustration and loss of motivation in the implementation of the program. A 42-year-old with Ph.D. in health administration claimed, "We should not expect something that took 20 years to be established in the
UK to only take 2-3 years here..... The program has to be adjusted to suit our needs with an accurate and precise plan." He added, in the UK, this program has shaped the clinical system and it should be the same here as well.

Moreover, some participants expressed the need for reforming the organizational culture and stressed that transformation of the culture is a fundamental step. A 49-yearold nurse complained, "Here, nurses are expected to do everything; and other health care providers, especially physicians and faculty members, do not play any role in the implementation of the program. Thus, the organizational culture should be modified to enhance the execution of quality improvement programs. "

\section{Inadequate financial resources}

Lack of sufficient financial resources was mentioned by all 25 participants. One of the participants suggested, "There has to be a specific budget set aside for workshops, staff training, and overtime pay [related to the implementation of the program]. When necessary costs are not budgeted for, things don't move forward as they should."

A 43-year-old participant with Ph.D. in health and clinical services added, "In hospitals, we say 'safety first', but the one who is responsible for implementation says we do not have enough money to buy 'Decosept' .... We have problems buying a set of patients' bed guards.... It doesn't matter if you work hard or not, your pay is the same."

5. Insufficient knowledge of the personnel and the management

According to the participants, one of the necessary measures for the implementation of the program is providing information to all involved parties about the program. In this regard, a 46-year-old head nurse explained, "When your hospital officials are not fully aware of the details and conditions of the program, how are they going to inform the rest of the personnel and monitor the proper implementation of the program? At the beginning, the personnel and the management should have been extensively trained, and not just by a short workshop."

\section{Inappropriate monitoring and evaluation}

According to many participants, many years have passed but standard performance-based indicators for the program have not yet been established. A 40-year-old nurse commented, "Now, instead of checking to see whether the right processes are followed or not, the existence or nonexistence of devices are checked and reported.... A monitoring and assessment team may evaluate us and give us a score, and a moment later, another team may come up with a very different score.... Many auditors, for the sake of the director [personal consideration] or other reasons, make their evaluations based on their own personal opinion.... Therefore, a number of individuals should receive specialized training to perform professional audits."

\section{Lack of coordination among various departments}

Many of the participants believed that separating clinical governance implementation from other management processes was a major obstacle to establishing the program. A specialist physician commented in this regard, "This program must become part of the hospitals' administrative and financial system.... The problem is that this program is like 
an 'isolated island' and has nothing to do with the administrative and financial systems.... It should be like the accreditation program to which everyone is attracted because of its financial resources; this program also needs to link up to financial and administrative systems; otherwise, it stays at where it is today."

On the other hand, according to some of the participants, it is necessary that all quality improvement programs be integrated and implemented as a single program. A 45-yearold supervisor remarked, "Nurses are confused and do not know if they have to take the clinical governance seriously or the accreditation program [another program that has recently been introduced in the hospitals around the county]." She added that some places have to keep up with ISO standards (for quality management) which make things even more complicated, and for this reason, none of the programs are implemented properly or completely.

8. Deficiencies in policies and procedures

For many participants, predefined clear procedures are necessary to guide the proper progress of the program. A 44 years old nurse argued, "Things will not change unless everyone feels invested in the program and until there is strict enforcement of policies and procedures.... There must be specific procedures in each department and everything should be documented."

9. Incomplete registration system and inadequate documentation

Many of the participants identified lack of proper infrastructure and adequate facilities as one of the barriers to implementation of clinical governance. A 48-year-old general practitioner noted, "Until we have accurate and integrated hospital information systems and proper facilities, we cannot achieve accurate and precise results from the patients' data."

\section{Quantitative Study}

From the 78 experts in the quantitative study who completed the questionnaires and returned them, 43 were female and 35 males, with the age range of 34-61 years. Their fields of study were the same as the participants in the qualitative study. They had between 9-26 years of experience in their occupation. Of them, 25 were the individuals who participated in the qualitative study as well. The participants in this part of the study rated the importance of the 9 extracted themes. The obtained scores for each of the themes, in order of highest to lowest, are displayed in Table 2.

\section{Discussion}

This study aimed to identify the barriers and their respective importance for the establishment of clinical governance program in teaching hospitals of Shahid Beheshti University of Medical Sciences in Tehran. In this study, 9 themes were extracted which were prioritized based on the rating of 78 experts who rated the barriers on a Likert scale from 1 to 5 . The average scores for all items were more than 4 , which indicate their importance in the implementation of clinical governance.

Findings of our study demonstrated a significant gap in the implementation of all 7 pillars of clinical governance in the investigated hospitals. According to the participants in this study, the most important obstacle in the implementation of the program was weak organizational leadership. They believed that to increase the interest of the implementers in the progress of the program, they should be given more authority. Participants noted that implementation of such an extensive program should not have been rushed as it did. Several national and international studies have stressed the importance of directors' commitment and support for the establishment of quality assurance systems to improve the efficiency and effectiveness of hospitals (1518). It seems that the improper selection criteria for the top management and their lack of sufficient understanding of the principles of clinical governance are the main reasons for their inefficient leadership.

The second most important obstacle that most participants noted was manpower deficiencies. According to the participants, a designated group of people with clear job description should be responsible only for implementation of clinical governance. They insisted that assigning tasks related to clinical governance to people with other responsibilities should be avoided. In addition, participants stated that the personnel with lower education may be less effective and prevent the proper execution of the program. They believed that the quantity and quality of human resources play an important role in the establishment of clinical governance. This finding is consistent with most research in the field of clinical governance. For example, Rashidian (2009) reported that supply of resources, including human resources, was limited which prevented complete implementation of the program (6).

Various national and international studies pointed out the need for adequate manpower to improve the quality of services $(17,19,20)$. Also, in some of the hospitals around the

Table 2. Priorities of the extracted obstacles and barriers to implementing clinical governance

\begin{tabular}{|c|c|c|c|c|c|}
\hline Priorities & Themes & Mean & Standard deviation & Minimum & Maximum \\
\hline Priority \#1 & Weak organizational leadership & 4.76 & 0.43 & 4 & 5 \\
\hline Priority \#2 & Insufficient human resources & 4.72 & 0.50 & 3 & 5 \\
\hline Priority \#3 & Inappropriate organizational culture & 4.70 & 0.54 & 3 & 5 \\
\hline Priority \#4 & Inadequate financial resources & 4.58 & 0.54 & 4 & 5 \\
\hline Priority \#5 & Insufficient knowledge of personnel and the management & 4.50 & 0.58 & 3 & 5 \\
\hline Priority \#6 & Inappropriate monitoring and evaluation & 4.42 & 0.57 & 3 & 5 \\
\hline Priority \#7 & Lack of coordination among various departments in the organization & 4.38 & 0.54 & 3 & 5 \\
\hline Priority \#8 & Deficiencies in policies and procedures & 4.36 & 0.53 & 3 & 5 \\
\hline Priority \#9 & Incomplete registration system and inadequate documentation & 4.32 & 0.50 & 2 & 5 \\
\hline
\end{tabular}


country, the number of personnel is even less than the national standards set by the Ministry of Health $(21,22)$. On the other hand, some national studies identified personnel's lack of knowledge about the program as a major problem for establishing the program (23) and empowering staff as one of the most important prerequisites for quality improvement programs (24), which are consistent with the findings of this study. Shortage of manpower, which is rooted in financial challenges and cost reduction, seems to be a common problem in many places around the world.

The third identified barrier on the priority list was inappropriate organizational culture. Most participants suggested improper organizational culture as major obstacles for carrying out the program. For instance, they argued the frequent change of management at all levels (planning, executive and administrative) cause's periodic changes in implementation priorities which are a source of delay for proper establishment of the program. In a study by Johnston et al, lack of a comprehensive plan and proper organizational culture were identified as the major barriers to implementation of clinical governance (25). Mehrabian et al named organizational culture as the most important component for improving efficiency (26). Afshar suggested that stability in management is tied to improvement in hospital performance (15) and Mohammadi argued that for successful and pervasive quality management, cultural reform is necessary (24). In another study, Mobasher et al showed that some aspects of organizational culture, especially those related to physicians and high-level managers, need improvement (27). All of these studies are in line with the current study and indicate fundamental concerns about organizational culture.

Another finding of this study was the barrier of insufficient funds which was rated as the fourth priority. From participants' perspective, lack of designated, continual and sufficient financial resources was considered an important obstacle in the implementation of clinical governance. The need for proper funding has been mentioned in many other studies. In a study by Rashidian et al, on the implementation of clinical governance in Tehran University of Medical Sciences, funding was cited as an area demanding attention (6). In many international studies, lack of resources, especially financial resources, has also been named as one of the main obstacles to the successful establishment of clinical governance $(19,25,28)$.

Staff and management's lack of knowledge about the program was ranked fifth in the list of barriers. Almost all participants in this study believed that this issue required prompt attention. According to the participants, if staff and management are not aware of the program's goals and objectives, they would not comprehend its importance and will be confused and ineffective in its implementation. A study in Baghiyatollah hospital in Tehran indicated that physical space, facilities, and equipment were not consistent with the Ministry of Health standards in that hospital (29), which could be the result of management's lack of knowledge about the basic requirements of the program. On the other hand, several studies have emphasized the positive effect of training of doctors, staff and administrators on improving the quality of services, and implementation of clinical governance (30-33). This is in line with the findings of this study. Perhaps, the lack of specific standards for the selection and periodic evaluation of the management and their lack of adequate and updated knowledge of management strategies are the main problems.

Inadequate monitoring and evaluation was rated as the sixth important barrier in this study. According to the participants, in addition to the lack of standard indicators for monitoring and evaluation, the monitoring and evaluation agents are not adequately trained and make most of their decisions based on the taste of the personal. In his study, Yarmohammadian concluded that indicators are basic tools for monitoring and evaluation (34). He argued that indicator development is one of the most important responsibilities of the management. Furthermore, Arab demonstrated that the current evaluation in private hospitals is more realistic and reliable compared to public hospitals (35). He added that the current evaluation systems require revision and reform. All these points indicate weaknesses in monitoring and evaluation of the health care system in the country.

Lack of coordination between different departments was ranked to be the seventh priority. The participants argued that clinical governance program should be integrated and coordinated with evaluation and feedback systems, performance-based financial reward and promotions criteria, and accreditation criteria. Otherwise, clinical governance could be viewed as a program without administrative and financial support. Mousavi, in his study, stated that to honor patients' rights, which are one of the components of clinical governance, an integrated, synchronized, and complete information system should be established (36). Furthermore, a genuine coordination between the patients' health information systems with other departments should exist to plan and implement the program properly. This is in line with the finding of this study.

Deficiencies in written policies and procedures were ranked as the eighth most important barrier. According to the participants, there must be strictly reinforced regulations and adequate standards to optimize the manpower. Under strict rules and regulations, all parties at all levels would fully cooperate with each other and decisions based on personal taste are eliminated. If there are strict regulations, guidelines are specific and the same for everyone. Furthermore, since one of the basic mechanisms of the clinical governance program is to have standard guidelines and documentation, previous and contextual experience should be used to prepare these guidelines. Van Kasteren et al discovered that guidelines are rarely followed. They suggested that to increase adherence to the guideline, appropriate, standard, and updated guidelines should be in place (37). Perhaps rushed implementation of clinical governance in Iran did not allow enough time to develop appropriate guidelines and procedures.

The ninth and final barrier identified in this study was incomplete recording and documentation system. According to some participants, to document medication and treatment information, to have necessary data to follow-up the patients, and to properly use all the hospital information, it is essential to have a hospital information system (HIS) that 
connects all departments. Proper equipment for the establishment of HIS is also necessary. In line with this finding, a study in one of the hospitals in Tehran concluded that HIS has a significant and positive role in increasing accuracy, speed, and ease of clinical procedures, and enhancing the quality of hospital services (38).

Since no study was found to have prioritized barriers, in this study, it was not possible to compare priorities with other studies. However, due to financial constraints and limited resources of health service providers in many places all around the world, prioritizing barriers seemed necessary for proper allocation of the limited funds and resources which was performed in this study.

Although studies report performance and quality improvement in some areas after implementation of clinical governance $(39,40)$, several obstacles to the establishment of this program were also reported in other studies around the country. These obstacles included lack of knowledge, lack of supportive culture and organizational factors, inadequate training, lack of commitment from the management, insufficient facilities, inadequate human resources and supervision, which are consistent with the findings of this study (41-43). A significant correlation was also stressed between proper planning for implementation and the complete and efficient establishment of clinical governance (44). In addition, adequate knowledge of top management, supporting culture, especially from top management and sufficient resources, were mentioned as main factors for proper establishment of the program (45).

One of the limitations of this study was that participants, instead of stating their experiences of the establishment of the program, answered the questions based on their own knowledge. This was addressed by reminding the participants during the interviews to use their own personal experiences and requesting real examples for their statements.

\section{Conclusion}

The findings of this study indicated that there are several barriers to implementation of clinical governance in hospitals of Shahid Beheshti University in Tehran. These barriers in order of importance were as follow: weak organizational leadership, insufficient human resources, inappropriate organizational culture, inadequate financial resources, insufficient knowledge of personnel and the management, inappropriate monitoring and evaluation, lack of coordination among various departments in the organization, deficiencies in policies and procedures, and incomplete registration system, and inadequate documentation.

For the proper establishment of the program and achieving its goals, policymakers and high-level managers should provide the needed means and resources, especially in the area of human resources, financial resources, and training of all personnel. Their full commitment and support for the implementation of the program are essential for achieving the ultimate goals of the program.

Furthermore, it is important to note that strategies to tackle the identified obstacles, particularly priorities of these strategies, were not addressed in this study. Future studies, using experts in this field, should seek to identify these strategies and prioritize them, so that specific steps for implementation of clinical governance program could be defined.

\section{Acknowledgements}

This article has been extracted from the thesis written by Dr. Abbas Ziari in Shahid Beheshti University of Medical Sciences, School of Medicine (Registration No: 274).

\section{Conflict of Interests}

The authors declare that they have no competing interests.

\section{References}

1. Health Do. A first-class service, quality in the NHS. London: Department of Health; 1998.

2. Shakeshaft A. A study of the attitudes and perceived barriers to undertaking clinical governance activities of dietitians in a Welsh National Health Service trust. J Hum Nutr Diet. 2008;21(3):225-38.

3. Health Do. The new NHS: modern, dependable. London: The Stationery Office; 1998.

4. Iran Mohameo. Circular 388044. Tehran, IranR2009/11/1. (Persian document).

5. Clinical Governance Instructions. In: Clinical Governance Office, Deputy of treatment, editors. tehran: Shahid Beheshti University of Medical Sciences; 2011 (Persian document).

6. Rashidian A. Clinical governance in Tehran University of Medical Sciences and quality improvement of health services. J Hosp. 2009;8(5):27-32. (Persian).

7. Adel A, Sadeghi A, Zarandi B, Moaref A, Zahmatkesh H, editors. Evaluation of clinical governance progress in an educational hospital in Tehran. National Conference of Quality Improvement with Clinical Governance Approach; 2011; Khorasan Shomali University of Medical Sciences. [Abstract] (Persian document).

8. Karimi M, Adel A, Raoofi S, Moraveji M, editors. Assess of barriers of clinical governance implementing of the personnel viewpoint of Mashhad University of Medical Sciences Hospitals. First National Conference of Clinical Governance 2011; Tabriz University of Medical Sciences. [Abstract] (Persian document).

9. Radpeiman P, editor Designing of quality improvement virtual education package with clinical governance approach. National Conference of Quality Improvement with Clinical Governance Approach; 2011; VKhorasan Shomali University of Medical Sciences. [Abstract] (Persian document).

10. Vaezi Z, editor An Experience of clinical governance implementing. National Conference of Quality Improvement with Clinical Governance Approach; 2011; Khorasan Shomali University of Medical Sciences. [Abstract] (Persian document).

11. Ghanati E, editor Challenges of clinical governance implementation in hospitals. National Conference of Quality Improvement with Clinical Governance Approach; 2011; Khorasan Shomali University of Medical Sciences. [Abstract] (Persian document).

12. Speziale HS, Streubert HJ, Carpenter DR. Qualitative research in nursing: Advancing the humanistic imperative: Lippincott Williams \& Wilkins; 2011

13. Graneheim UH, Lundman B. Qualitative content analysis in nursing research: concepts, procedures and measures to achieve trustworthiness. Nurse Educ Today. 2004;24(2):105-12.

14. Morse JM. Qualitative Nursing Research a Contemporary Dialogue. Newbury Park: Sage Publications; 1991.

15. Afashar Kazemi MA, Salarifar M, Nasiripoor A, Manoochehrimogadam Z. Survey of effective management factors in Tehran's Heart Center. J Hosp. 2011;10(2):1-9. (Persian).

16. Anbari Z, Sedghiani E, Tabibi J. A comparison among Arak's hospitals about productivity mechanisms of logistic services. J Adv Med Biomed Res. 2005;13(52):49-56. (Persian).

17. Delgoshaie B, Maleki M, Dehnaveie R. Evaluation of essential requirements for implementing of quality management in Rasoul Akaram hospital. Health Monitor. 2004;3(3):211-7. (Persian).

18. van Hamersveld KT, den Bakker E, Nyamtema AS, van den Akker T, Mfinanga EH, van Elteren M, et al. Barriers to conducting effective obstetric audit in Ifakara: a qualitative assessment in an under- 
resourced setting in Tanzania. Trop Med Int Health. 2012;17(5):652-7.

19. Campbell SM, Sheaff R, Sibbald B, Marshall MN, Pickard S, Gask L, et al. Implementing clinical governance in English primary care groups/trusts: reconciling quality improvement and quality assurance. Qual Saf Health Care. 2002 Mar;11(1):9-14.

20. Delgoshaie B, Tabibi J, Pahlavan P. The model of improving human resource in Iran's health system. Res Medicine J. 2010;31(4):317-25. (Persian).

21. Farajzadeh Z, Nakhaee M, Tabiee S, Nasiriforg A, Pejmankhah S. The study of number and structure of nursing personnels in Birjand University of Medical Sciences. Mod Care J. 2006;3(3):5-9. (Full Text in ersian).

22. Sadeghi far J, Poorreza A, Ahmadi B, Zeraati H, Arab M. Assessment of human resource needs in Ilaam University of Medical Sciences Hospitals. J Ilam Uni Med Sci . 2011;19(1):24-31. (Persian).

23. Vatankhah S, Gohari M, Abdi Z. The relationship between organizational culture and improvement of quality improvement model in Tehran's selected hospitals. Health Monitor. 2010;9(2):189-95. (Persian).

24. Mohammadi A, Shoghli A. The gap between expectation and perception of Zanjan's hospitals personnels about determinants of pervasive management. J Adv Med Biomed Res. 2006;14(54):30-9. (Persian).

25. Johnston G, Crombie IK, Davies HT, Alder EM, Millard A. Reviewing audit: barriers and facilitating factors for effective clinical audit. Qual Health Care. 2000 Mar;9(1):23-36.

26. Mehrabian F, Nasiri poor A, Keshavarzmohammadian S. The study of importance of identified determinants of human resource productivity in Guilan University of Medical Sciences personnels' viewpoint. J Adv Med Biomed Res. 2011;19(75):94-106. (Persian).

27. Mobasher M, Nakhaee N, Garoosi S. Evaluation of organizational culture in Kerman's educational hospitals. J Med Ethics Hist Med. 2007;1(1):45-52. (Persian).

28. Wallace LM, Freeman T, Latham L, Walshe K, Spurgeon P. Organisational strategies for changing clinical practice: how trusts are meeting the challenges of clinical governance. Qual Health Care. 2001 Jun;10(2):76-82.

29. Bahadori M, Mirhashemi S, Panahi F, Tofighi S, Zaboli R, Hosseini $\mathrm{SM}$, et al. The survey of structure, processes and activities in Baghiatallah hospital's emergencies wards. J Mil Med. 2007;4(9): 25763. (Persian).

30. Mohsenpoor L, editor Nursing management skills teaching, a step to clinical care quality improvement. First National Nurses Meeting of the Army Force; 2011; Tehran. [abstract] (Persian document).

31. Narenjiha M. Patients satisfaction in Qods clinic in Tehran. Iran J Med Educ. 2012;12 (1):77-85. (Persian)

32. Rabiee F, Moaiedi N, Naderi Z, Ali abadi frahani K, Shamsi M. The outcome of in-service education in the viewpoint of university experts. Educ Methods J. 2011;4(2):85-9. (Persian).

33. Yousefi A, Rezaie A. Continues education for physician, the basis of heath service quality improvement. Iran J Med Educ. 2000;1(1): 15-9. (Persian).

34. Yarmohammadian M, Yaghoobi M, Momikhani J, Ansari M, Karimian J, Kiani M, et al. Indicators development, the most essential source for human resource evaluation in heath system. Health Inf Manag. 2010;7:546-55. (Persian)

35. Arab M, Poorreza A, Zeraati $\mathrm{H}$, Ravangard R. Is there any relation between evaluation grade and the operation of Tehran University of Medical Sciences Hospitals?. J Manag Inf Health. 2005;2(1):1-7. (Persian).

36. Mousavi A. The role of clinical governance in realization of patients' rights. J Med Ethics. 2011;5(18):161-78. (Persian).

37. van Kasteren ME, Kullberg BJ, de Boer AS, Mintjes-de Groot J, Gyssens IC. Adherence to local hospital guidelines for surgical antimicrobial prophylaxis: a multicentre audit in Dutch hospitals. J. Antimicrob. Chemother. 2003 Jun;51(6):1389-96.

38. Roozbahani R, Mozaffarian B, Poordizaji MK. The outcome of using hospital integrated information system in improving service quality in Masih Daneshvari Hospital. Payavard-Salamat. 2012;6(2):128-37. (Persian).

39. Davoodi R, Soltanifar A, Rahmani S, Sabouri G, Asadi M, Zare Hoseini M, et al. Clinical Governance: Efficacy of Establishment in Mashhad Hospital. J Patient Saf qual. 2014;2(1):48-52.

40. Gauld R, Horsburgh S. Measuring progress with clinical governance development in New Zealand: perceptions of senior doctors in 2010 and 2012. BMC Health Serv Res. 2014;14(1):547.

41. Dehnavieh R, Ebrahimipour H, Jafari Zadeh M, Dianat M, Noori Hekmat S, Mehrolhassani MH. Clinical Governance: The Challenges of Implementation in Iran. Int Hosp Res. 2013;2(1):1-10.

42. Ravaghi H, Rafiei S, Heidarpour P, Mohseni M. Facilitators and Barriers to Implementing Clinical Governance: A Qualitative Study among Senior Managers in Iran. Iran J Public Health. 2014;43(9):126674.

43. Ravaghi H, Zarnaq RK, Adel A, Badpa M, Adel M, Abolhassani N. A Survey on Clinical Governance Awareness Among Clinical Staff: A Cross-Sectional Study. Glob J Health Sci. 2014;6(6):p37.

44. Aryankhesal A, Tabatabaee S, Kalhor R, Kakeh-mam E, Joyani Y, Moradi N. Association of organizational climate and hospital performance in establishment of clinical governance in Tehran University of Medical Sciences (2012). J Qom Uni Med Sci. 2014;18(5):43-50. (Persian).

45. Ravaghi H, Heidarpour P, Mohseni M, Rafiei S. Senior managers' viewpoints toward challenges of implementing clinical governance: a national study in Iran. Int J Health Policy Manag. 2013;1(4):295. 